\title{
Precision Agriculture Robot for Seeding Function and Leaf Disease Detection
}

\author{
Lokanadam J K S Sai Ganesh ${ }^{1}$, Mohith N Raate ${ }^{2}$, \\ Nithin $\mathrm{T} \mathrm{N}^{3}$, Pavan $\mathrm{G}^{4}$ \\ ${ }_{1,2,3,4}$ Under Graduate Students, Dept. of Electronics and \\ Communication Engineering, \\ Dr. Ambedkar Institute of Technology, India
}

\begin{abstract}
Agriculture, allied with various sectors has been the largest livelihood provider for India. It also contributes to about $15-17 \%$ of the total Gross Domestic Product (GDP) of India. There is a need to decipher the issues like controlled use of pesticides, proper leaf disease detection. The automation of agriculture has emerged as a futuristic technology. This implementation of precision agriculture concept will lead to revolutionary change in the domain of agriculture. The automation concept helps the farmers in increasing the gain from the soil and saving time. Automation has been able to lower the energy required for performing physical tasks. Improvement in the quality of life of the farmers and reducing heavy labor and tedious tasks is the main aim of this work. A prototype of the precision agriculture robot is presented which is designed for performing the functions like ploughing, seeding, sowing, water sprinkling and pesticide spraying function. The robot is a four wheeled module controlled by LPC2148 Microcontroller. The robot also inhabits a leaf disease detection module based on advanced image processing and machine learning which will be used to detect the type of disease affected. Further, suitable pesticide is sprayed to the affected part of the plant depending on the type of disease.
\end{abstract}

Keywords - Precision Agriculture; Leaf Disease Detection; Autonomous Agriculture Robot, Controlled pesticide spray

\section{INTRODUCTION}

Generally, all the repetitive agriculture tasks are being done either by the use of man power or heavy machinery. Several primitive seed sowing methods such as animal drawn funneling, pipe drilling and drilling using tractor were being used. The above mentioned techniques require labour, a lot of time and energy consumption. Usage of heavy machinery results in exposure to high level noise and vibration affecting the health and work performance of the farmer. The key factors kept in mind during the development of autonomous field robots are:

- $\quad$ Speed

- Energy Efficiency

- Accuracy

By using this robot technology, one can sit in a cool and comfortable place and can accomplish various tasks by just monitoring the robot.

Agriculture is not all about growing crops but also taking care of the crops and preventing it from various diseases and threats. If the plant gets affected it directly affects both man-kind and animal life. Therefore, detection of the disease is an important and urgent task. One of the traditional methods of plant disease detection

\author{
Prof. Nithyashree $\mathrm{S}^{5}$ \\ ${ }^{5}$ Assistant Professor, Dept. of Electronics and \\ Communication Engineering, \\ Dr. Ambedkar Institute of Technology, \\ India
}

is by observing the plant with the naked eye, but this does not give accurate results as the disease can be microscopic which is not visible to the naked eye. This problem can be solved by automating the monitoring process by use of advanced image processing technique and machine learning. The proposed work aims in making the automated system easily available for the farmers using the device for early detection of disease in the plants.

India is a country whose economy mostly depends on agriculture. Farmers have variety of options to cultivate crops in the field, yet the cultivation of crops and harvesting of crops is done in old and traditional manual way which may lead to improper management, in turn leading to reduction in the yield. The yield can be increased and quality can be improved by the use of technology.

\section{LITERATURE SURVEY}

In 2014, Sunitha M has carried out seeding robotics for the irrigation system. Some of the major problems in the Indian agricultural are rising of input costs, accessibility of skilled labors, lack of water resources and crop monitoring. She concluded that the major problems in India can be overcome by automating agriculture with robots. The automation in the agriculture would play a very important role in reducing their efforts [1]. Later in 2015, M Priyadarshini has found on the robot which performs operation like soil, moisture testing, seeding, spraying pesticides, removes compost from the field, which also performs obstacles avoidance operation and metal detection in the path. The robot was designed using a DTMF technique embedded in the cell phone. The DTMF technique overcomes the problem of using Bluetooth or RF module at longer distances which have limited working range. The integrated system of Agribot which is capable of performing activities like seeding, weeding, spraying of fertilizers and insecticides communicate with other robot using the Wi-Fi. The robot was controlled by Arduino Atmega2560 controller. A sophisticated Raspberry pi minicomputer was used to control and monitor the working of robot. The robot has a hexapod body which enables the robot to move in any desired direction as required. The robot was embedded with a ultrasonic proximity sensor in order to overcome the obstacles in the path, and an underbody sensor system was installed to check if the seed is planted or not. The robot digs a hole in soil, plants the seed and covers the 
hole again with soil. The necessary pre-emergence fertilizers are applied on it and move on along with communicating with another robot near to it using Wi-Fi. Command based self-guided digging and seed sowing rover, a sensor guided rover for digging, precise seed positioning and sowing were proposed in order to lower the human effort and increase yield efficiency [2]. Ankit Singh, Abhishek Gupta, Akash Bhosale, Sumeet Poddar in 2015 did a research was focused on rover's navigation is performed by remote guiding devices fortified with the positioning system. An Arduino Atmega2560 controller and an ultrasonic radar sensor are used to avoid the obstacles. The robot was controlled using wireless module which is controlled by PC/TAB/Mobile. This module sends an acknowledgement message regarding the seed tank to the farmer. The agriculture robot performs only two operations that include digging (ploughing and sowing) and cover the plough area with soil. The seed dropped using a stepper motor and a spike wheel is used to dig a hole [3]. Firthous Begum, P. Vignesh in 2013 gave the motivation of the research is to decrease harvesting cost and increase the productivity. Conventional harvesting methods involve intensive labor and are inefficient considering both time and economy. Machine harvesting systems may provide a partial solution to overcome these issues by efficiently removing the fruits from the trees. Hence a total production cost of over $35-45 \%$ of harvesting can be reduced. The main of designing agribot is to reduce the cost of harvesting [4]. Buniyamin N, Wan Ngah W.A.J, Sariff N, Mohamad Z in 2011 concluded that Mobile robot path planning has a few main properties according to type of environment, algorithm and completeness. The properties are whether it is static are dynamic local or global and complete or heuristic. The static path planning refer to environment which contains no moving objects or obstacles other than a navigating robot and dynamic path planning refers to environment which contains dynamic moving and changing object such as moving obstacle [5].

\section{METHODOLOGY}

The proposed system is based upon ARM Microcontroller, Wi-Fi module, DC motors, Relays, Camera and Image Processing. The peripherals are interfaced to the microcontroller to perform various operations like ploughing, seeding, leveling, water spraying and pesticide sprinkling. The system is controlled by Zigbee and Wi-Fi module from Android smart phone. The wireless communication is used to move the robot in four directions as front, back, right and left. The microcontroller in the proposed model enables various functions in the field according to the commands received from smart phone. Agriculture robot is also capable of performing Leaf Disease Detection using the SVM Classifier.

\subsection{Robot Module:}

The robot module is built using DC motors, Communication Module, Relays and their driver circuits, Battery and Microcontroller. The module has two sections: robotic section and control section, which intercommunicate with each other. The robotic section inhabits ploughing unit, drill to dig the soil, seed dispenser, and seed storage. The robotic components are controlled by control section inhabiting motors, microcontroller, and power supply.

The robot module is powered with a battery. The robot is provided with $12 \mathrm{~V}$ battery to operate the system. The base frame consists of four wheels connected to four arms and the rear wheel is driven by DC motor. At an end of the frame, cultivator is attached which is driven by dc motor which is made to dig the soil. The seeds are dropped through drilled hole on the shaft by the linked mechanism with soil digging. A leveler is used to close the seeds and water pump sprayer is used to spray the water.

The robot is guided and controlled by the remote guiding device. When the motors are initiated, the module moves in the programmed columns of ploughed land. It then performs the functions of digging the soil and sowing the seeds. The microcontroller gives the orders to all the networks, and responds accordingly to the data processed by the programs for the factors collected.

\subsection{Disease detection module}

Digital camera is integrated to the field robot. The field robot goes through the field and captures the images of the leaves. The captured plants are then classified as diseased and non-diseased. The affected part of the diseased plants is then captured using the camera. The functions of preprocessing, transformation and clustering are performed on the captured images. Then, the processor is input with the processed images and compared with the images initially trained images. The automatic pesticide sprayer sprays the pesticide to the localized area in the leaf if the image captured is the image of the affected part. The robot will move further and the processor discards the image if the image is not an image of affected part. After the evaluation of the diseases all the identified data and the suitable actions need to be taken is sent as alert to the centralised cloud for storage and the farmer's smartphone in the form of SMS for necessary response.

In order to spray the pesticide to the affected part, an automatic pesticide sprayer is involved. The system consists of a sprayer filled with required pesticide. The sprayer ensures a continuous flow of pesticide. The sprayer adjusts the height in order to spray the pesticide on the affected part of the plant.

\subsection{SVM:}

SVM is a supervised learning algorithm in which the processing is done by dividing into two phases. The first processing phase is the offline phase or Training Phase. In this phase, a set of input images of leaves (diseased and normal) are processed by image analyzer and certain features are extracted. Then these features will be given as input to the classifier, and along with it, the information whether the image is that of a diseased or a normal leaf. The classifier then learns the relation among the features extracted and the possible conclusion about the presence of the disease. Thus the system is trained. 
In the proposed system, MATLAB is being used for the image processing. An image processing for disease detection is performed as follows:

STEP 1:- Initially, the image is captured in $\mathrm{YCbCr}$ form and then it is converted into RGB.

STEP 2:- The HSV image is obtained by converting the RGB image. HSV is abbreviated as Hue, Saturation and Values. The color space is described in terms of colors (hue or tint), shade (saturation or amount of gray) and their brightness value.

STEP 3:- The Hue image is later obtained by converting the HSV image.

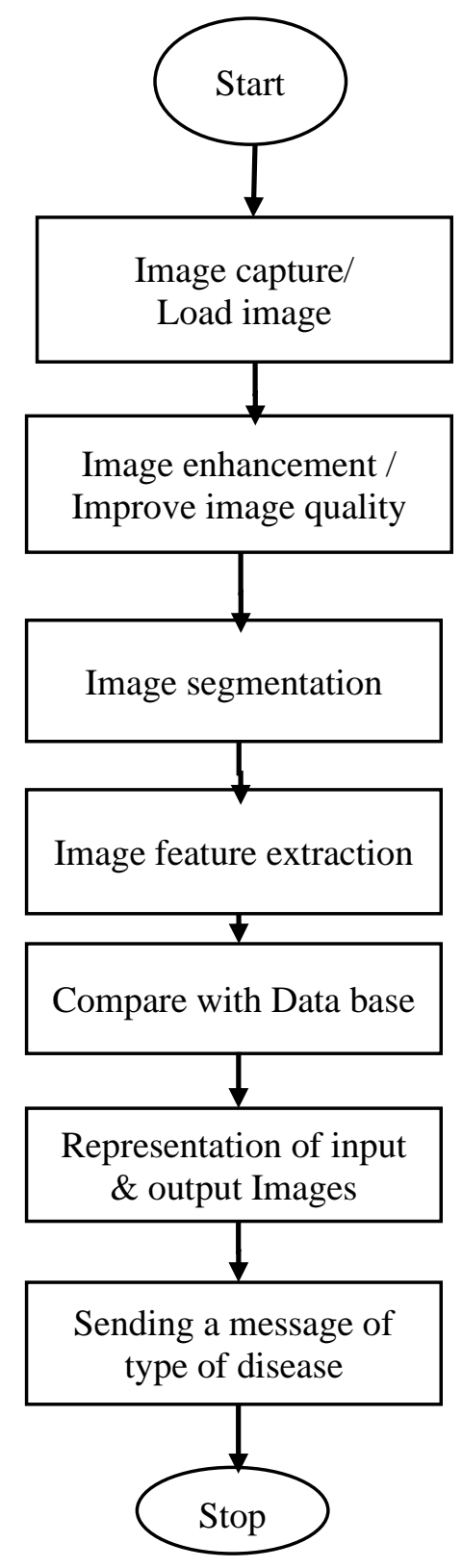

Fig 2 : Flowchart of Leaf Disease Detection Technique

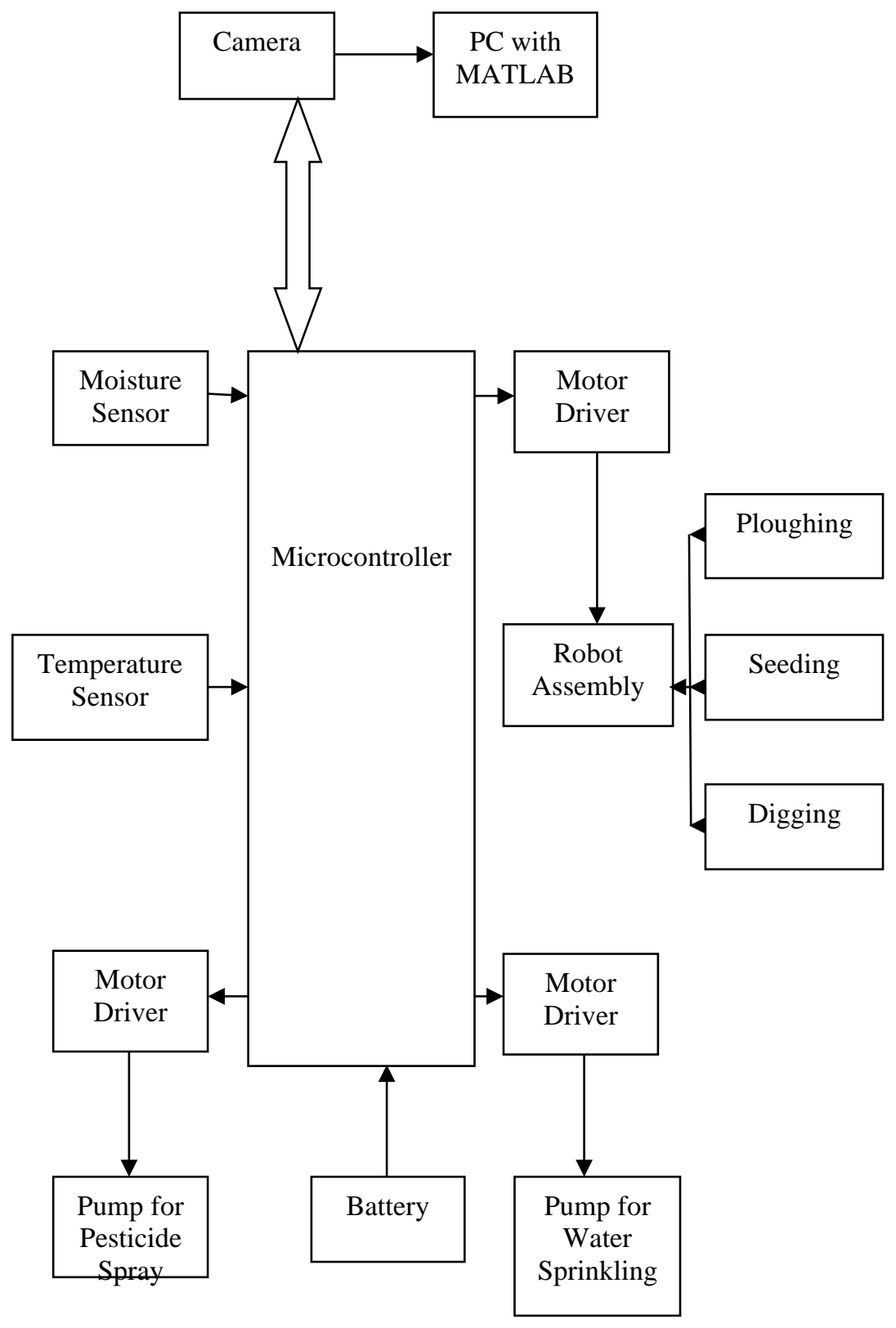

Fig 3: Block Diagram of Robotic Module

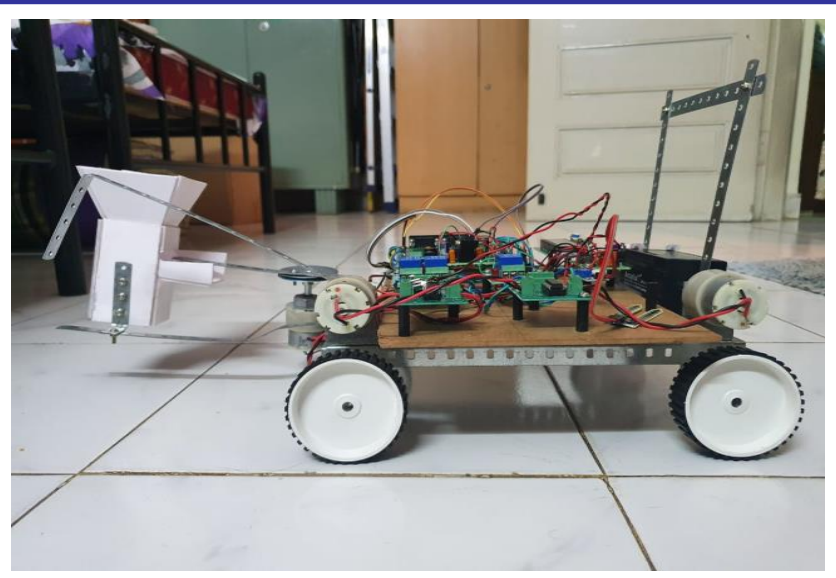

Fig 1: Prototype of Proposed Model 


\section{RESULT AND DISCUSSION}

The robotic module performs functions: Ploughing, Seeding, Digging and Water Sprinkling. These functions are performed with the aid of sensors. The sensors (moisture and temperature sensors) collect the data from the surroundings. The robotic module processes the provided data and determines the amount of water to be sprinkled. The robot then performs ploughing. The seed to be sown are loaded into the module. The depth to be dug and the distance to be placed are pre-designed depending on the type of seed used. The robot movement is achieved by the DC motors controlled by the software module.

A pump for sprinkling of pesticide is installed in the module. The module is initially trained to create different databases of leaves to compare with the captured image. The image is captured by the leaf detection module and is classified using Support Vector Machine classifier. The type of disease found in leaf is then determined and the same is displayed. The type of pesticide to be used for the particular disease is loaded into the module and the robot sprays the pesticide to the affected leaf.

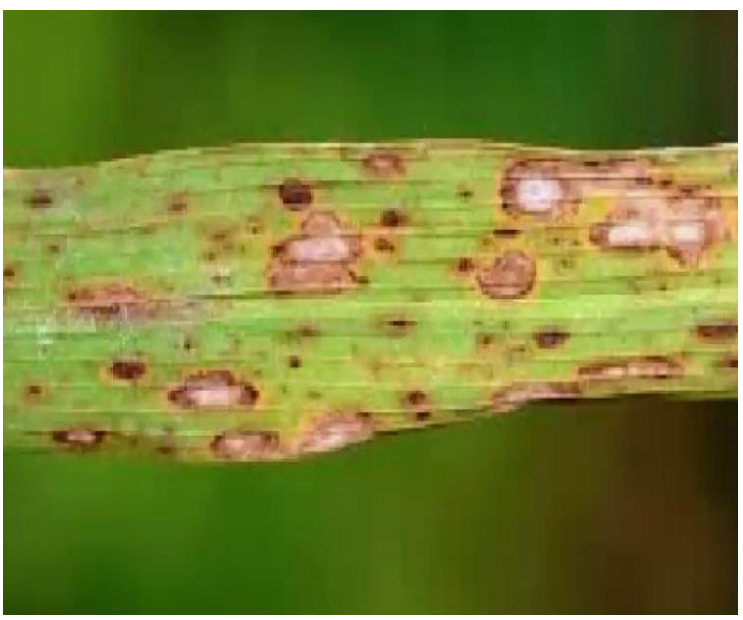

Fig 4: Diseased Leaf Image of Paddy

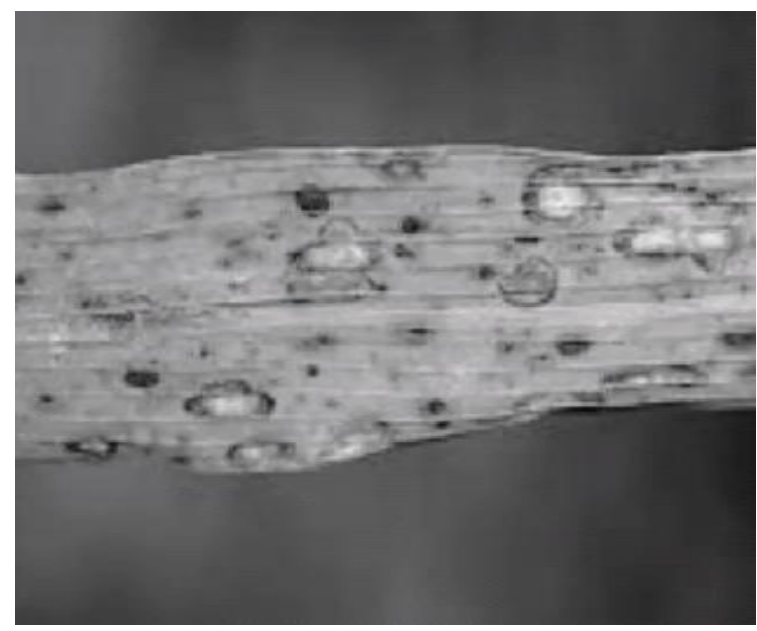

Fig 5 : Gray scale image of input image

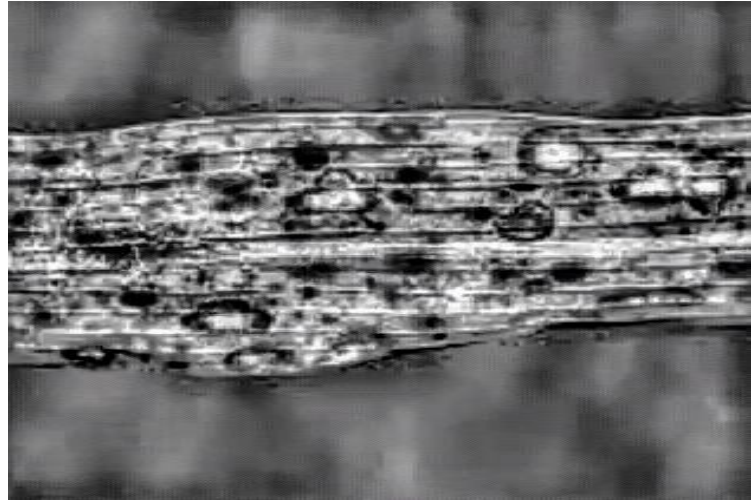

Fig 6: Adaptive Histogram image of input image

\section{CONCLUSION}

The agriculture robot was proposed based on the automation of agriculture. The proposed method helps the farmers to easily perform their agricultural activities and efficient leaf disease detection. The robot reduces the stress and strain suffered by the farmer. Information about the leaf disease was obtained through image processing algorithms. Image processing offers a noninvasive approach to detect disease. It was found that with this algorithm, an efficient detection was obtained. Hence, it reduces the problem of wrong determination of disease.

\section{FUTURE WORK}

In the proposed system, the leaf disease detection was only confined to a specific disease in the proposed system. The number of diseases to be identified can be extended for more diseases in future with the development of the robot. With the development of science and technology, the accuracy and precision of this agriculture robot can be enhanced by adding additional equipments.

\section{ACKNOWLEDGEMENT}

We would like to thank our Principal Dr. C. Nanjundaswamy, Head of the Department, Dr. Ramesh $\mathrm{S}$, our co-ordinator Dr. Tanuja P and my guide Asst. Prof. Nithyashree $\mathrm{S}$ for their valuable advice and technical assistance.

\section{REFERENCES}

[1] Sunitha .M, "Seeding Robot", The Intl. Conf. on Information, Engineering, Management and Security 2014 (ICIEMS 2014)

[2] M. Priyadarshini, L. Sheela, "Command Based Self-Guided Digging and Seed Sowing Rover", International Conference on Engineering Trends and Science \& Humanities (ICETSH-2015)

[3] Ankit Singh, Abhishek Gupta, Akash Bhosale, Sumeet Poddar, "Agribot: An Agriculture Robot", International Journal of Advanced Research in Computer and Communication Engineering Vol. 4, Issue 1, January 2015.

[4] N. Firthous Begum, P. Vignesh, "Design, and Implementation of Pick and Place Robot with Wireless Charging Application", International Journal of Science and Research.

[5] Buniyamin N., Wan Ngah W.A.J., Sariff N., Mohamad Z, "A Simple Local Path Planning Algorithm For Autonomous Mobile Robots", International Journal Of Systems Applications, Engineering \& Development Issue 2, Volume 5, 2011.

[6] Y Nikhil Kumar, C H Haswanth, M Hima Kiran, M Koteshwar Rao, Rahul Raj, Gopi Krishna Saramekala, Proma Anonya Chakrobarthy, "Automated Sedd Sowing Agribot", 2019 IEEE $1^{\text {st }}$ International Conference.

[7] Sahiya Sidheek, Tinu Varghese, Unnimaya Biju, Robin George, Anu Mathew, Tina Abraham, "Multipurpose Agricultural Robot (AGRIBOT)", IJAREEIE-2019. 